\title{
Anti-LGR5 Monoclonal Antibody BNC101
}

National Cancer Institute

\section{Source}

National Cancer Institute. Anti-LGR5 Monoclonal Antibody BNC101. NCI Thesaurus. Code C126794.

A humanized monoclonal antibody targeting leucine-rich repeat-containing G-protein coupled receptor 5 (LGR5), with potential antineoplastic activity. Upon administration, the anti-LGR5 humanized monoclonal antibody BNC101 targets and binds to LGR5, thereby inhibiting LRG5-mediated signal transduction pathways. This prevents proliferation of LRG5-expressing tumor cells. LGR5, a member of the Wnt signaling pathway, is a cancer stem cell (CSC) receptor overexpressed on certain cancer cells; it plays a key role in CSC proliferation and survival. 